\title{
A Kind of Calibration Device for Bitumen Needle Forcemeter
}

\author{
LiuLu, Hongbo Guo, Yishu Zhou and Genqiang Jing \\ Research Institute of Highway Ministry of Transport, No.8 Xitucheng Rd., Beijing, China
}

\begin{abstract}
According to "The vertification regulation of bitumen needle forcemeter for road"(JJG 067-2006), calibration of bitumen needle forcemeter is resolved by measuring indication device and time controller respectively. But this way of calibration can't solve the problem of starting indication device at the same time touching off time controller. There is still a time controller starts lagging phenomenon in most of the calibrated instruments. Measured time interval is inaccurate and penetration value is not accurate. Using the embedded multichannel integration measurement technique, the calibration device of bitumen needle forcemeter is realized, and the accuracy of the test results is guaranteed.
\end{abstract}

Keywords-mechanical structure; measure; penetration value; asphalt; calibration device

\section{INTRODUCTION}

Penetration value is the representation method of bitumen consistency, reflect the rheological properties of asphalt, and express degree of hardness and softness for asphalt. Bitumen needle forcemeter is a kind of penetration value determining key instrument for pavement petroleum asphalt, polymermodified binder, and liquid petroleum pitch distillation or emulsified asphalt after evaporation residue ${ }^{[1]}$. According to the national standard 'asphalt penetration measurement'(GB/T 4509-2010) ${ }^{[2]}$, penetration value is determining. Penetration value is showed in a standard needle straight in a certain load, temperature, time to wear into the depth of the asphalt samples. The time interval showed by time controller, standard needle inserted depth of asphalt sample, temperature and constant temperature time, standard needle roughness, surface tension, are the factors influencing penetration value test value size ${ }^{[3]}$. Timing device according to the time and standard needle inserted depth of asphalt sample is to determine the penetration test values directly index, the size of only the measured values of the two indicators are accurate, to ensure the reliable of penetration value.

According to 'The vertification regulation of bitumen needle forcemeter for road'(JJG 067-2006),the traceability of value for bitumen needle forcemeter is solved by calibrating indication device, standard needle, needle pitman, time controller, and temperature control devices. In the use of bitumen needle forcemeter remains found inaccurate phenomenon of penetration value test results. Analysis of the causes of discovery indication device and time controller can also trigger is the cause of the phenomenon of the main problems. When the indication device of bitumen needle forcemeter has started, but time controller does not work, the time interval of bitumen needle forcemeter is not accurate, the penetration value is certainly not accurate. According to the working principle of bitumen needle forcemeter, a kind of calibration device for bitumen needle forcemeter is researched. An embedded multi-channel integrated measurement technology is used in bitumen needle forcemeter calibration device. Calibration for bitumen needle forcemeter can ensure the accuracy of penetration value test results.

\section{Problems of Existing CALibration MethoD}

The bitumen needle forcemeter is calibrated in the transportation sector, since 'The penetration test apparatus of petroleum bituminous for road'(JJG 067-2006) ${ }^{[4]}$ published. The calibration scheme of time controller and indication device, shown follows:

\section{A. Calibration of Indication Device}

Adopt the method of visual inspection to calibrate the division value of bitumen needle forcemeter. Lock the needle pitman at a fixed position. Adjust the base platform to the level state. Between the needle pitman and the surface of the base respectively into the $1.00 \mathrm{~mm}, 5.00 \mathrm{~mm}$ and $10.00 \mathrm{~mm}$, $20.00 \mathrm{~mm}$ and $40.00 \mathrm{~mm}$ five fourth gage block. Adjust the lifting device to make the needle pitman contact with the top of gage block gently. Zero the display device. Remove the gage block. Hold down the release button of needle pitman. Make the needle pitman contact with the top of base platform gently. Record indicating value of the display device respectively. Run each test three times and take the mean to eradicate any discrepancies. Calculate maximum permissible errors between indicating value of the display device and standard length of gage block. The division value of bitumen needle forcemeter is $0.1 \mathrm{~mm}$. The maximum permissible errors is \pm 1 penetration value, that means $\pm 0.1 \mathrm{~mm}$.

\section{B. Calibration of Time Controller}

Remove standard needle from bitumen needle forcemeter. Lock the needle pitman. Release time respectively in the $5 \mathrm{~s}$ and $60 \mathrm{~s}$ two gears. At the same time start the instrument release switch and standard timing stopwatch. Write down indicator of the stopwatch, when the instrument display reaches $5 \mathrm{~s}$ (or $60 \mathrm{~s}$ ) standard of instantaneous stop timing stopwatch. Run each test three times and take the mean to eradicate any discrepancies. The maximum permissible error calculation of needle pitman release time should be in line with the requirements of $\pm 0.1 \mathrm{~s}$. 
Analysis of the existing calibration methods for bitumen needle forcemeter $[5,6,7]$, there are three problems.

(1) Indication device and time controller are calibrated respectively in vertification regulation. This calibration method can be ensure the accuracy of displacement value and run time of standard needle. But simultaneity between indication device and time controller can't be calibrated. Can't ensure that bitumen needle forcemeter at the same time of needle pitman began to decline, time controller starts timing immediately. This leads to asphalt penetration is still not accurate measurements, the penetration value measure can't correctly. Test results can't correctly reflect the consistency of asphalt, affect the quality of road construction.

(2)The existing bitumen needle forcemeter calibration method can't be real-time, visual display the test data of indication device and time controller, can't reflect asphalt penetration value reproducibility.

(3)In the process of calibration for indication device, the gauge block removed, needle rod quickly began to free fall. The process will cause the needle pitman and the base have certain collision damage.

\section{Design for Recurrence Set of Penetration Value}

Repeatability, stability and reproducibility are important measurements of measuring appliance characteristics. Similarly, reproducibility is vital for bitumen needle forcemeter calibration. In the original calibration method, needle pitman free fall and falling distance is very short, increased the difficulty of the movement time monitoring. To solve the above problems, use $45^{\circ}$ wedge slider (as shown in figure 1) to convert the vertical freefall to horizontal motion. Horizontal rail (as shown in figure 2) is configured as horizontal sliding track of wedge slider. Place $45^{\circ}$ wedge slider on the horizontal rail. Recurrence set of penetration value is place on the base of bitumen needle forcemeter. The bottom of needle pitman is contacted with the bevel of $45^{\circ}$ wedge slider. Press down the release button of bitumen needle forcemeter, and make needle pitman free fall. Run each test ten times. The test results are shown in table I.
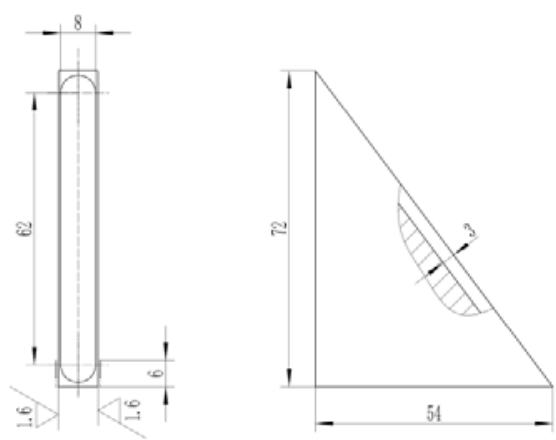

FIGURE I.

$45^{\circ}$ WEDGE SLIDER
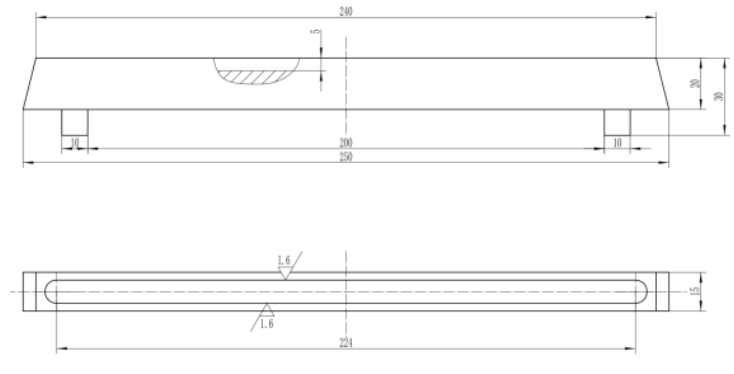

FIGURE II. HORIZONTAL RAIL

TABLE I. WEDGE SLIDER PENETRATION VALUE RECURRENT EXPERIMENT

\begin{tabular}{|c|l|}
\hline Times & Result \\
\hline 1 & can slide \\
\hline 2 & can't slide \\
\hline 3 & can't slide \\
\hline 4 & can't slide \\
\hline 5 & can't slide \\
\hline 6 & can't slide \\
\hline 7 & can't slide \\
\hline 8 & can't slide \\
\hline 9 & can't slide \\
\hline 10 & can't slide \\
\hline
\end{tabular}

Two reasons why the wedge slider can't slide horizontally:

(1)The wedge slider is too heavy;

(2)The sliding surface between wedge slider and horizontal rail is not smooth enough.

For the above reasons, we improve the design scheme. The quality of needle pitman is $47.5 \mathrm{~g}$, needle pitman freefall gravity

$F=m g=47.5 \times 10^{-3} \times 9.8 \mathrm{~kg} / \mathrm{m}^{3}=0.47 \mathrm{kN}$. First of all, decomposing the force along the wedge edge direction and vertical direction of the wedge edge, $F_{1 \mathrm{v}}=0.47 \mathrm{kN} / \sqrt{2}=0.33 \mathrm{kN}$, secondly decomposing the force above along the vertical and horizontal directions, $F_{2 h}=0.33 \mathrm{kN} / \sqrt{2}=0.235 \mathrm{kN}$. Therefore, when the friction force of the wedge slider is less than $0.235 \mathrm{kN}$, the wedge slider can realize the horizontal sliding.

\section{Overall Design of Calibration Device}

Existing calibration method of bitumen needle forcemeter has problems, for example, simultaneity between indication device and time controller can't be calibrated, measured values can't be visible displayed and recurrent, collision damage 
during calibration process. So we design a new calibration device of bitumen needle forcemeter. We use embedded multichannel integrated measurement technology to solve the Simultaneity problem between indication device and time controller, and show the measured value in the display device. In addition, we use the wedge slider and the horizontal rail to convert the vertical displacement which is hard collected to horizontal displacement, then we collect data using range device. The new calibration device of bitumen needle forcemeter includes four parts: display device, range device, wedge slider and horizontal rail. The hardware diagram is shown in Figure III and technical solution process is shown in Figure IV.

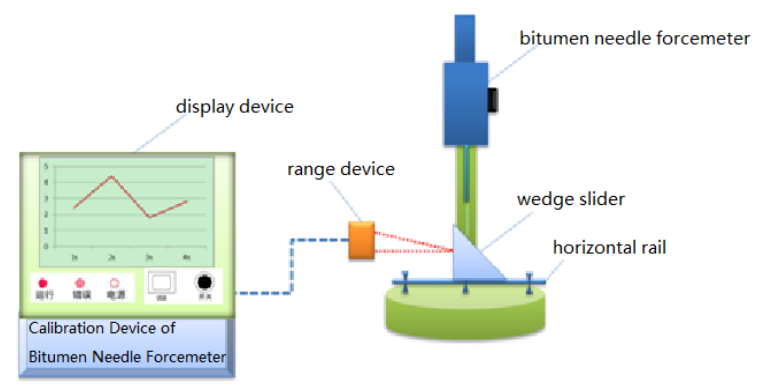

FIGURE III. HARDWARE DIAGRAM

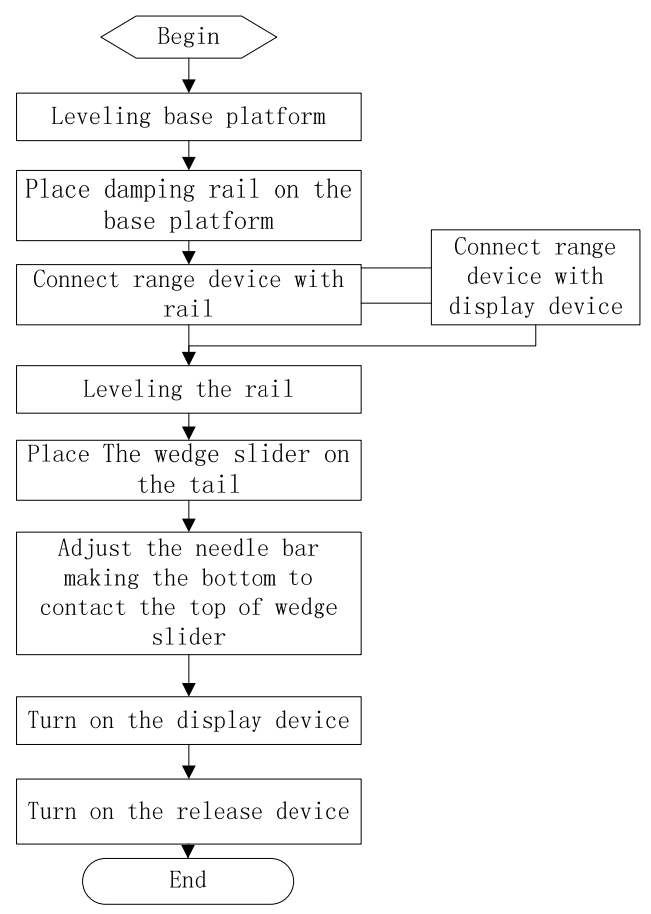

FIGURE IV. TECHNOLOGY SOLUTION PROCESS

Installation step:

(1)Level the base platform of bitumen needle forcemeter.

(2)Place horizontal rail on the base platform.

(3)Connect one end of range device with adjustable damping rail, at the same time, connect the other end with display device.
(4)After leveling the damping rail, place the wedge slider on the adjustable damping rail.

\section{CONCLUSION}

Current calibration method provided by " The vertification regulation of bitumen needle forcemeter for road" (JJG 0672006) has problems that simultaneity between indication device and time controller can't be calibrated, measured values can't be visible displayed and recurrent, collision damage during calibration process. Regarding the issue above, this paper research an new calibration device of bitumen needle forcemeter. The main conclusions are as follows.

(1)The penetration value recurrent device composed by wedge slider and horizontal rail can make penetration value recurrent.

(2)The less quality of wedge slider, the less friction between wedge slider and horizontal rail, which can convert the vertical displacement to horizontal displacement.

(3)The new calibration device of bitumen needle forcemeter can solve the existing problems.

\section{REFERENCES}

[1] Chen Bainian. Testing Technology for Road Engineering Measurement Uncertainty Lecture-Reasonable Evaluation of Using Bitumen Needle Forcemeter to Test Measurement Uncertainty of Bitumen Penetration Value [J]. Communications Standardization, 2004, (5):26-29.

[2] GB/T 4509-2010, Test Method of Bitumen Needle Forcemeter[S].

[3] Zhu Jian, Xu Haiyu. Discussion On The Factors Affecting Bitumen Penetration Value[J]. Xinjiang Association for Quality Inspection First Symposium Papers.

[4] JJG067-2006, The Vertification Regulation of Bitumen Needle Forcemeter for road [S].

[5] Zuo Xinjian. Several Issues On The Bitumen Needle Forcemeter Standard. China Measure, 2010, (11):97.

[6] Gao Bingmei, Pei Jianjun, Wang Xiumei, Yao Dehong. Influence of Bitumen Penetration Value Testing Conditions on Penetration Index [J]. Petroleum Bitumen, 2006,20(4):62-64.

[7] Yang Junchi. Automatic Bitumen Needle Forcemeter Routine Calibration and Troubleshooting[J]. China Science and Technology Information, 2005,(1):66. 